\title{
Flúor: límites permisibles en el agua de consumo humano e ingesta adecuada recomendada
}

\author{
Fluorine: permissible limits in water for human consumption \\ and recommended adequate intake
}

Edgar García-Torres ${ }^{1 a}$

Fernanda Elizabeth Rodríguez-Rodríguez ${ }^{1 \mathrm{~b}}$

Correspondencia: edgar.garcia@ujed.mx
D https://orcid.org/0000-0002-7799-4352

D https://orcid.org/0000-0003-2202-8533

\section{Estimado Editor:}

Hemos leído el artículo de revisión titulado "Flúor y fluorosis dental" de Martínez-Cántaro $\mathrm{N}$ et al., ${ }^{1}$ publicado en su importante Revista Odontológica Basadrina (2021;5(1):75-83). Claramente, es un artículo de revisión de suma importancia odontológica que pretende sintetizar los beneficios y desventajas de la exposición al flúor, a través de sus diferentes formas, y el impacto que se tiene sobre la salud humana, principalmente en la forma de fluorosis dental. Queremos felicitarlos y a la par hacer algunas contribuciones.

El flúor (F), nombre que proviene del latín fluere «fluir», descubierto por el químico Carl Wilhelm Scheele en 1771 y aislado en 1886 por el ganador del Premio Nobel de Química en 1906, Henri Moissan, nos hace alusión, por su etimología, a sus características físicas: un gas corrosivo de coloración amarillo-pálido. Además, es un elemento químico que, en la tabla periódica, conforma el grupo VIIA correspondiente a los halógenos, siendo el más electronegativo y con una importante condición para atraer fuertemente a otros electrones, lo que le confiere mayor reactividad y toxicidad. ${ }^{2}$

Es importante establecer que el $\mathrm{F}$ no está catalogado como un nutriente esencial, ya que no participa de manera primordial en el desarrollo y crecimiento del organismo humano; además, su baja o nula ingesta no condiciona, de ninguna manera, el correcto desarrollo de las estructuras a las que se incorpora cuando es ingerido, tales como el cemento dental, la dentina, el esmalte dental y/o los huesos. ${ }^{3}$

Aun cuando el $\mathrm{F}$ no se considera nutriente esencial, se ha indicado una ingesta adecuada (IA) para diferentes grupos etarios, tras el único efecto benéfico del fluoruro en la dieta sobre la prevención y gravedad de la caries. En general, se recomienda una IA de fluoruro proveniente de todas las fuentes, tanto para niños como para adultos, establecida de $0.05 \mathrm{mg} / \mathrm{kg}$ de peso corporal por día. En la Tabla 1 se ejemplifica la IA propuesta por la Autoridad Europea de Seguridad Alimentaria (EFSA, por sus siglas en inglés).

\footnotetext{
${ }^{1}$ Universidad Juárez del Estado de Durango. Facultad de Odontología. Durango, México

a Cirujano Dentista y Maestro en Ciencias Estomatológicas

${ }^{\mathrm{b}}$ Estudiante de pregrado
} 


\section{Tabla 1}

Ingesta adecuada recomendada de fluoruros en diferentes grupos de edad (Ingesta proveniente de todas las fuentes de F)

\begin{tabular}{ccc}
\hline \multirow{2}{*}{ Grupo etario } & \multicolumn{2}{c}{ Fluoruros $\mathrm{mg} / \mathrm{d}$} \\
\cline { 2 - 3 } & Hombres $^{* *}$ & Mujeres $^{* *}$ \\
\hline 7-11 meses & 0.4 & 0.4 \\
$1-3$ años & 0.6 & 0.6 \\
$4-6$ años & 1.0 & 0.9 \\
$7-10$ años & 1.5 & 1.4 \\
$11-14$ años & 2.2 & 2.4 \\
$15-17$ años & 3.2 & 2.8 \\
$\geq 18$ años & 3.4 & $2.9^{*}$ \\
\hline
\end{tabular}

\footnotetext{
* Ingesta recomendada en mujeres embarazadas y en periodo de lactancia.

** Calculado con los pesos corporales de cada grupo etario (ver referencia).

Nota. Tabla tomada y modificada de Journal EFSA, 2013. ${ }^{3}$
}

Con respecto a la exposición de $\mathrm{F}$ a través del agua de consumo humano, fue en la década de 1930 cuando se observó que comunidades con concentraciones de fluoruro en agua, de 0.7 a $1.2 \mathrm{mg} / \mathrm{l}$, presentaron menos prevalencia de caries que en comunidades con niveles inferiores de fluoruro en agua, destacando el efecto benéfico en el aumento de la resistencia a la caries en los niños. ${ }^{3}$ Sin embargo, actualmente, la Organización Mundial de la Salud, claramente estipula que la fluoración artificial e intencionada de agua para consumo humano debe encontrarse a una concentración final de entre un $0.5 \mathrm{mg} / \mathrm{l}$ y $1 \mathrm{mg} / \mathrm{l} .{ }^{4}$ Por otra parte, el Servicio de Salud Pública de EE. UU. (PHS, por sus siglas en inglés) establece un límite de fluoración intencionada de aguas de consumo de $0.7 \mathrm{mg} / \mathrm{l} .{ }^{5}$ En lo que respecta al nivel de fluoruros en el agua de mantos acuíferos y agua subterránea, naturalmente no exceden los $10 \mathrm{mg} / \mathrm{l}$, aunque es bien conocido que en diversas partes del mundo (India, China, África Central, América del Sur, América del norte) pueden encontrarse niveles elevados de este elemento; el nivel natural más alto del que se tiene reporte es de $2800 \mathrm{mg} / \mathrm{l} .{ }^{4}$ Es así que la OMS establece valores de referencia para la cantidad de fluoruros en el agua de consumo humano de $1.5 \mathrm{mg} / .{ }^{4}$ Todas estas recomendaciones, sobre el límite permisible de fluoruros en agua de consumo y en la ingesta adecuada recomendada, tienen dos principales objetivos; por un lado, hacer presentes los efectos anticariogénicos del $\mathrm{F}$ y por el otro, evitar, en la medida de lo posible, el desarrollo de fluorosis dental (FD), como consecuencia de una sobre exposición a este elemento en etapas de desarrollo dental, tanto de dientes deciduos como permanentes. No obstante, se han encontrado alteraciones dentales en la forma de FD leve en concentraciones entre $0.9 \mathrm{mg} / \mathrm{l}$ y $1.2 \mathrm{mg} / \mathrm{l}$ de fluoruro en agua de consumo. ${ }^{4}$ Por lo anterior, es fundamental conocer los límites actuales de consumo permisibles para este elemento; además, adoptar y divulgar las recomendaciones y directrices sobre el uso de fluoruro para la prevención de caries, con énfasis en la población pediátrica. ${ }^{6}$

Reconocemos el efecto anticariogénico del $\mathrm{F}$, pero consideramos necesario precisar que los máximos beneficios, y los menores efectos adversos del F, se obtienen tras su utilización de manera tópica y en dientes erupcionados, y que la presencia de caries dental no es únicamente por una deficiencia o baja ingesta de F, esta implica una interacción de factores como una mala higiene dental, dietas ricas en carbohidratos y susceptibilidad a su desarrollo, por mencionar algunos. 
Finalmente, es importante destacar que la ingesta de sal fluorada, el uso de enjuagues fluorados, la aplicación de gel y barnices fluorados, así como el uso de tabletas comprimidas de F, solo estaría justificado en los lugares donde no se realiza la fluoracion controlada e intencionada de agua de consumo, y deben evitarse en aquellos lugares donde los niveles de $\mathrm{F}$ en el agua sobrepasen los valores de referencia estipulados por las instancias de salud correspondientes.

\section{Referencias}

1. Cántaro NY M, Pereyra YM, Catacora LAC, Torres ERM, Laura AA, Nina MSC. Flúor y fluorosis dental. Revista Odontológica Basadrina. 2021;5(1), 75-83.

2. Ullah R, Zafar MS, Shahani N. Potential fluoride toxicity from oral medicaments: A review. Iran J Basic Med Sci. 2017;20(8):841-848. doi:10.22038/IJBMS.2017.9104

3. EFSA PANEL ON DIETETIC PRODUCTS, NUTRITION, AND ALLERGIES (NDA). Scientific opinion on Dietary Reference Values for fluoride. EFSA Journal.2013;11(8):3332.

4. Guidelines for drinking-water quality: fourth edition incorporating the first addendum. Geneva: World Health Organization; 2017. Licence: CC BY-NC-SA 3.0 IGO

5. U.S. Department of Health and Human Services Federal Panel on Community Water Fluoridation. U.S. Public Health Service Recommendation for Fluoride Concentration in Drinking Water for the Prevention of Dental Caries. Public health reports (Washington, D.C.:1974). 2015;130(4), 318-331. doi:10.1177/003335491513000408

6. Toumba KJ, Twetman S, Splieth C, Parnell C, van Loveren C, Lygidakis NA. Guidelines on the use of fluoride for caries prevention in children: an updated EAPD policy document. Eur Arch Paediatr Dent. 2019 Dec;20(6):507-516. doi: 10.1007/s40368-019-00464-2. 